\title{
27 A VIRTUAL ENTERPRISE MODEL, AS PROPOSED WITHIN PROJECT ICIV AIMED AT SUPPORTING NETWORKED SMES
}

\author{
Roberto Tononi - ENEA, Italy \\ Nicola Marturano - SINTESI, Italy
}

\begin{abstract}
In Italy, on national and EU funding, ENEA has launched Project ICIV (Concurrent Engineering for the Virtual Enterprise) to set up service centers for SME's.

These centers will be promoting aggregations of SME's based on a Virtual Enterprise model. The paper provides an outlook of the model and some insights on major organizational and technical problems.
\end{abstract}

\section{INTRODUCTION}

In Italy, in the framework of an R\&D program aimed at experimenting new business models and operational tools for SME's, ENEA (national institution for research on Energy, Environment and Innovation) has launched project ICIV (Concurrent Engineering for the Virtual Enterprise) funded by the Italian Ministry of Research, the EU and ENEA itself.

One of the objectives of the project is that of developing and testing a model for aggregations of SME's, herein referred to as Virtual Enterprise (V.E.), aimed at supporting them in the effort to operate in the global market, covering all phases of the product/service life cycle.

The model envisages groups of SME's as stable entities which activate different enterprises depending on the changing market demands.

\section{THE ARCHITECTURE PROPOSED IN ICIV FOR NETWORKED SME'S}

The basic philosophy, followed in grouping SME's in project ICIV, is that of keeping the single enterprises as autonomous as they are willing to be.

\footnotetext{
- Corresponding author address: ENEA, CR Casaccia, Via Anguillarese 301, 00060 S.M. di Galeri (RM), Italy, tel: (++39 6) 30486151, fax: (++39 6) 30486168, e-mail: tononi@casaccia.enea.it
} 
To this extent, levels of hierarchy in the organizational structure are to be kept to a minimum; that is the reason which has led to the choice of a hub and spoke architecture, depicted in the following schema:

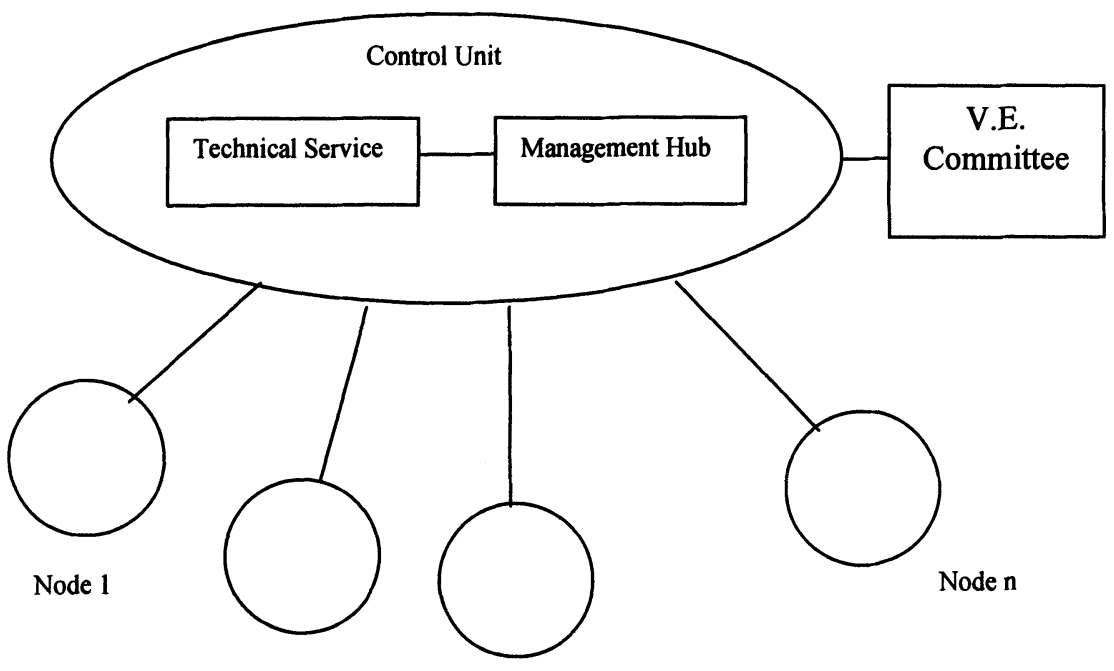

Node 2

Node 3

Figure 1 - ICIV Architecture

The network is featured by:

- a number of nodes - the grouped SME's, all with the same rights, each one seen as an autonomous provider of service/product towards the Virtual Enterprise;

- a hub node - the Control Unit, which has the function of coordinating the operation of the Virtual Enterprise;

- the V.E. Committee, whose members are the representatives of the grouped SME's, which makes decisions on long term strategies of the Virtual Enterprise.

\section{The nodes}

The SME's which are grouped in the V.E. can be complementary with each other, with the purpose to cover the entire life cycle of the V.E. output, as well as additionable, i.e. providing the same kind of product/service, with the purpose to increase the productive capability of the V.E.

The relationship between nodes and V.E. is of non-exclusive nature : each node is engaged in the V.E. with a fraction of its entire productive capacity and is allowed to maintain its own business.

When an SME joins the V.E. , the contractual agreement states for the above fraction

- quantity of the product/service to be supplied on request of the V.E.,

- timing related to the availability of this supply, 
- quality features of the supply,

- cost structure of the same supply;

reference to the above factors is made as maximum quantity, quality levels to be guarantied, maximum cost; the time factors are seen as upper bounds.

In other words, the expected supply of each node is "prepackaged" when the SME joins the V.E.; at that time, there will be some negotiation, but basically the SME makes its choices on how it intends to be engaged in the V.E.

This prepackaging implies that the impact, on the SME operation, of a call from the V.E. , will be foreseeable and limited to the extent decided upon by the SME itself.

This approach tends to favor the internal optimizations of the node, whereas it actually limits the optimization range of the whole V.E. tender; this trade off is dictated by the need of ensuring an acceptable level of reliability of the V.E. operation, which, otherwise, can turn to be an Achilles heel of the network of independent enterprises. In practice, each node is seen as a production center, which is asked for predefined services; in addition, the node organizes and manages autonomously its internal operation in order to meet the V.E. requests. It can be noted, here, a similarity with the object-oriented approach to software development.

Likewise, the prepackaging allows the production plan for a V.E. tender to be defined through an automatic procedure as shown later in this paper, as if dealing with a real enterprise.

\section{The Control Unit}

Though the nodes may change over time, depending on the market demand and on the willingness of the SME's to join or leave the V.E., the Control Unit represents the stable part of the Virtual Enterprise.

In addition, though the autonomy of the grouped SME's is watched with care, the network cannot function without an appropriate coordinator.

The Control Unit functions are those to be provided for the whole aggregate of nodes and have been sorted out to be assigned to two separate, though tightly correlated, units: the Management Hub (Mgm Hub) and the Technical Service (TS).

The Mgm Hub is in charge of the following functions:

- Marketing management;

- Implementation of the directions of the V.E. Committee;

- Interface of the V.E. with the procurer;

- Project Management;

The Mgm Hub cares the development of the marketing strategy for the V.E. as a whole, identifying the target market of the V.E. and the marketing mix to be offered to it. However, the marketing strategy is submitted to the approval of the V.E. Committee. Within the approved strategy, the Mgm Hub defines the promotion plan of the V.E..

In addition, the Mgm Hub, on the basis of the market picture collected by the Technical Service, develops the framework of new initiatives which can be advantageously undertaken by the V.E.

Wherever these new initiatives can be implemented with the current resources of the V.E., the Mgm Hub is free to undertake all the actions which are necessary to 
turn the initiatives into projects, such as getting in touch with the potential procurers to acquire knowledge of their wants and needs.

However, if a new initiative requires extra resources for the V.E., the Mgm Hub has to set up a business plan and submit it to the V.E. Committee for approval; in case of positive response, the Hub will move on in order to get new SME's to join the V.E.

In either case, the Mgm Hub acts as the proxy of the V.E. towards the potential procurer and is in charge of defining a draft tender; the draft will be turned into the final tender applying to it the Quality Function Deployment procedure, which will see the involvement of all nodes identified by the Mgm Hub as those to be activated to implement the tender.

As the V.E. proxy, the Mgm Hub negotiates with the procurer and signs the procurement contract.

In addition, the Hub defines the whole production plan, which meets the specifications included in the contract, and will be in charge of its implementation and control.

As mentioned above, whereas the medium term planning is assigned to the Hub, the long term planning (i.e. entering new markets and acquiring new resources) is performed by the Hub along with the V.E. Committee. Planning for the short term, i.e. for the activities each node is expected to perform in order to meet the production plan, is left to the single nodes.

The Mgm Hub also functions as the secretariat of the V.E. Committee, taking care of organizing its meetings and of processing its directions.

The Technical Service (TS) is in charge of the following functions:

- Collection of market information for the Mgm Hub and implementation of the promotion plan issued by the Mgm Hub;

- System Quality management for the V.E.;

- Configuration Management for each procurement;

- Communication network management;

- Provision of Concurrent Engineering tools to the nodes.

As to the first above function, the TS works as the marketing operation support of the Mgm Hub.

With regard to System Quality, the TS builds up the framework of practices and procedures to be followed by the V.E. as a whole.

In particular, the TS develops the "Quality Manual" for the V.E., consistently with ISO 10013, and submits it to the approval of the V.E. Committee.

It is also in charge of developing the quality implementation plans over the whole life cycle of the product/service provided by the V.E., as well as the appropriate process capability plans.

Also these plans are subjected to the V.E. Committee approval; following this approval, the TS supervises their implementations and reports to the V.E. Committee over violations to these plans and proposes corrective actions.

The TS carries out the auditing system of the V.E.; this system is devised so that to be kept as light as reasonable.

In fact, audits are performed by the TS on SME's which request to join the V.E.; these audits are aimed at assuring the quality level of the new prospective node and 
at determining any necessary adjustment. After joining, each node is marked, by the auditing system, in terms of a few attributes which are deemed to feature its quality of operation; later on, periodically, the TS only checks on these attributes and a new audit will be necessary only when facing relevant changes of these attributes.

The basic objective of the above system quality management is that of endowing the V.E. with the ISO $900 x$ certification, even without requiring that all nodes be certified.

As to the Configuration Management (CM) function, the TS is in charge of developing a generic $\mathrm{CM}$ plan which is submitted to the V.E. Committee approval; then, in each procurement, the generic plan is turned to a specific plan which defines the whole set of procedures to be followed in order to ensure the product/service integrity.

Within this context, basic duties of the TS are

- identify the appropriate documentation scheme and flow,

- collect and safely store the project documentation,

- manage the change proposals.

To this extent, the TS will identify, for each project, the Equipment Planning Diagram, the Configuration Items, the related Baselines and how to process the Engineering Change Proposals.

As to the last item, those change proposals, which implies modifications of the contractual specifications of deliverables towards the procurer, are to be reported to the Mgm Hub for the related renegotiations with the procurer.

With regard to the communication network, the basic specification, assumed in the V.E., is that the nodes should find on the network the typical services one usually gets on a Local Area Network, though the physical interconnections are implemented on a Wide Area Network resorting to equipment and services provided by public utility companies.

The above implies that the TS is in charge of:

- providing and maintaining the V.E. communication network;

- contracting with public utility companies as to the services to be acquired for the V.E. nodes;

- bulletin dispatching to the nodes, as to the information multicast by the Mgm Hub or other nodes;

- periodic inquiry on the work status of all active nodes and report to the Mgm Hub;

- Shared Data Base management and service;

- Multi-users synchronous audio-video conferencing;

- Providing E-mail service;

- Enabling electronic commerce activities between nodes and among the V.E. and its procurers.

From a physical point of view, a minimum requirement - hub and spoke configuration is achieved connecting each node to the TS through the ISDN; the available transmission rates are just as sufficient as to allow low-quality audio-video conferencing. More appropriate a solution is the one resorting to the ATM 
technology; however, at the time of this writing, this solution isn't available everywhere and at cost deemed acceptable by every SME.

In ICIV, e-commerce refers, as in most contexts, to a whole set of activities which include, on the one side, the delivery of promotional messages aimed at presenting the V.E. on the web and at establishing informal communication channels with either prospective or acquired clients or both, and, on the other side, the exchange of commercial documents- implementation of commercial transactions, such as ordering, invoicing, payment and the like: to this regard, EDI standards, such as UN/EDIFACT, will be implemented by the TS which will receive a message from the sender node, will encode it and pass it to the receiver node, so that to get around the difficulties each node would face in implementing this standard.

In addition, the TS keeps track of the procedures and practices of "Electronic Public Procurement" being set up and unfolded as directives of the EU Commission, in order for the V.E. to be ready to seize opportunities on the side of the requests for tenders by the european public institutions; to this extent, the TS also monitors the picture of the current requests.

Another relevant function of the TS is that of making available to the nodes a Concurrent Engineering tool kit, with the aim at :

- $\quad$ reducing the time to market;

- $\quad$ improving the quality of what is going to be produced by the V.E..

Both goals are consistent with the Concurrent Engineering approach; as a matter of fact "parallel" performing of activities tends to reduce the time to market; in addition, Concurrent Engineering practices force a close collaboration among nodes engaged in different phases of the product/service life cycle and that favors the identification of potential defects or inadequacies of the product/service in early phases of the production process, i.e. when corrective actions are easier to undertake and result in lower impact on total cost.

The set of tools foreseen within project ICIV includes:

- CAx , such as Computer Aided Design, Computer Aided Process Planning, Computer Aided Manufacturing, Material Requirements Planning, Computer Aided Storage and Transportation;

- DFx , such as Design For Manufacturing, Finite Element Analysis, Design For Assembly \& Disassembly, Design for Environment, Design For Reliability \& Maintenance, Logistic Support Analysis;

- Simulation and Virtual Reality visualization;

- Rapid Prototyping.

Basically, the TS makes available the above tools as operational infrastructures which allow the nodes to perform cooperative work.

The major operational links are established between design activities and down stream implementations, such as, for instance, the activities performed by the designer (Design For manufacturing) and those performed by the manufacturer (Computer Aided Manufacturing), though also later life cycle stages are taken care of, such as with Logistic Support Analysis.

The adoption of Concurrent Engineering sets the stage for the basic problem of the interoperability among the tools utilized by different nodes.

In ICIV this problem is tackled under two perspectives: 
- the collection and management of information about the components to be delivered by the various nodes;

- the exchange of services among nodes.

Within the first perspective, a solution to the problem is given resorting to the adoption of the standard STEP/ISO10303 and of the Shared Data Base for the V.E. as it is described, later on in this paper.

Within the second perspective the exchange of services is made manageable by the adoption of CORBA technology (Common Object Request Broker Architecture) as deployed by the Object Management Group (OMG); that will allow the application of a node to ask for computational services provided by applications of other nodes and get the related results, regardless of the utilized SW and HW platform and of the physical location on the network, and through the activation of mechanisms which are transparent to the nodes; such as, just to give an example, in the case of a designer node application asking the manufacturing node application for executing a simulation of the manufacturing procedure of a newly designed component.

It won't be unnoticed an inconsistency introduced by the Concurrent Engineering approach into the organizational mechanisms of the V.E. As a matter of fact, even though each node is seen as a service center to which "prepackaged" provisions are asked for, the interactions between nodes, triggered by the utilization of Concurrent Engineering tools, get in the way of a smooth and foreseeable flow of activities of each "interested" node; in other words, an interaction may act as a disturb for a node.

In addition, the introduction of concurrent engineering, into an aggregate of SME's, for sure, doesn't ease organizational problems.

The above, just to mention that not all the goodies come with the adoption of Concurrent Engineering and that the V.E. organizational aspects are to be designed with much care.

\section{The V.E. Committee}

It is kind of the equivalent of the Board of Directors of a real enterprise.

Its basic function is that of making decisions on what proposed by the Control Unit.

The Committee includes the representatives of all nodes which, then, must comply with its decisions.

Herein are listed the major occurrences which require a V.E. Committee approval or decision:

- issue of the marketing plan by the Mgm Hub;

- changes of the marketing plan;

- undertaking of new initiatives requiring new resources for the V.E.;

- new SME's joining the V.E;

- issue of the Quality Manual and the Quality plan by the TS;

- violations of Quality Implementation plan as reported by the TS;

- issue of the Generic Configuration Control plan by the TS;

- call for a Committee meeting by some of the nodes. 


\section{THE BASIC MECHANISM OF MEDIUM TERM PLANNING}

In ICIV, medium term planning refers to the definition of the "production plan" which occurs when, facing a potential procurement, the Mgm Hub tailors a tender.

The related mechanism is herein summarized (1) (2), since it may give a perspective on the V.E. operation, as conceived in ICIV.

- First, the supply required by the procurer is split into components deliverable by V.E. nodes (the MRP diagram or the Equipment Planning diagram, depending on the perspective).

- The quality level, of the supply required by the procurer, is translated into consistent quality levels of the above components.

- Only nodes capable of delivering those quality levels are then activated.

- There may be more than one node activated to deliver the same type of component; in this case, the quantity levels to be delivered by each of these nodes are computed as fractions, of the whole quantity, proportional to the rates of product/service subscribed by the nodes when joining the V.E.. For instance, when, in a specific procurement, the total quantity to be supplied for a type of component is 90 and where a node A subscribed to deliver 200 units, whereas node B subscribed for 100 units, node A will be called for providing 60 units and node $B$ for 30 units.

- Once identified the nodes to be activated and their quantitative levels of deliverable, the production plan is defined assuming, as the starting reference, the supply date asked for by the procurer; based on the timing each node has subscribed, as to its own capability of delivery, the production plan is defined proceeding backwards in time, down to marking the start date for the whole project. If the start date falls after the "current" date, the project can be undertaken.

The time span between current date and start date is left as an operational margin to the activated nodes.

What should be noticed on the above mechanism is that:

- quality levels, subscribed by the nodes, act as discriminators of the nodes to be activated;

- the nodes that commit for higher quality levels may count on higher engagements in the V.E. projects;

- both factors act as solicitors, the former for higher levels of quality, the latter for higher involvement in the V.E. ;

- the whole mechanism allows the Mgm Hub to develop, in a very short time, the production plan simply resorting to a CAPP SW tool (made available by the TS) using as inputs the procurement features and the data of commitment subscribed by the nodes when joining the V.E..

When dealing with procurements which are recurrent or which have a limited impact on the V.E. operation and are within the current marketing strategy, the Mgm Hub may go for the straight definition of the production plan and notify it to the activated nodes for confirmation.

For more complex or unfamiliar procurements, the output of the above process is handed as "preliminary", by the Mgm Hub, to the nodes to be activated in order to revise it applying the Quality Function Deployment procedure, the implementation of which is assisted by the TS. 
Nodes which violate their commitments with the V.E. are penalized in terms of down grading of their levels of involvement in the future projects; other nodes, which make up for these "failures", are up graded if they whish so.

\section{THE APPROACH TO INFORMATION INTEGRATION AND SHARING}

In order to manage the various life cycle phases and, in particular, to define a tender and the related production plan, it is necessary to compound the various pieces of information which describe the various components deliverable by the nodes; that is feasible if the pieces of information can be integrated with each other.

The integration is logically feasible if the information refer to common concepts in the application domain; it is physically feasible if the information are stored in compatible formats.

Both logical and physical compatibility can be attained compelling the nodes to supply their information in accordance to predefined conceptual schemes and physical formats; but this solution is definitely banished in the framework of the V.E. , as long as it implies a reformulation and a rearrangement of the information set owned by the nodes.

An alternative solution is that to translate the node's information to common conceptual schema and formats.

However this activity of translation cannot be left to the nodes and is provided by the TS as a common service for all nodes.

Within Project ICIV, the common conceptual schema and formats (3) are those derived from the standard STEP (STandard for the Exchange of Product model data) - ISO 10303.

The job of translation is assigned to the Technical Service to lighten the burden of the nodes and to rely on an operator which is fully familiar with the methodology of the standard.

The translation is actually a two-step process: first, the product logical description, as handed by the node, is to be mapped over the logical description, for the same product, foreseen by the standard (i.e. the "Application Protocol"); then the description is to be encoded in the standard physical format.

Needless to say, the first step is the more onerous, since it implies an effort of mapping the semantics used by the supplier node over the semantics foreseen by the standard for the product and the related context being considered. That requires some collaboration between Technical Service and node.

For some time to come, one can face a situation where no Application Protocol seems to be available for the product and/or the related context, in which case the standard description is to be framed according to the rules for developing new application protocols: yet another reason for assigning the management of product descriptions to the Technical Service.

After the mapping, the node native data set can be transformed into the (semantically) equivalent STEP data set (represented through the EXPRESS language) and then encoded into the STEP file format, a character-based on ISO 8859, for representation of characters using 8-bit encoding. The use of characterbased encoding means that STEP files are portable across all major operating 
systems and computing environments and can be transferred across networks using e-mail or EDI.

Through the process of translation, the nodes can exchange information even though they use different description systems for their products; in fact, a description, sent form node $A$ to node $B$, is first translated from node $A$ system to the STEP file format and then, from this, is translated back to the node B system where the information is fully understood; of course, the two translation processes will be different.

But, in ICIV, STEP is not only used to obtain direct communications among nodes.

As a matter of fact, within STEP, one finds an interesting part called SDAI (Standard Data Access Interface) ISO 10303-22, which establishes a standard process on how to store STEP data (formulated in EXPRESS) into a database; this last one can be of whatever type as long as its related DBMS provides for an SDAIlike interface.

With this provision, the node native data set can be translated to STEP data and then, as opposed to being formatted through a character-based encoding suitable for exchange, can be stored into a database. To an external viewer, these data appear to conform to the data model specified in the STEP Application Protocol, as if they were stored in EXPRESS format.

ICIV resorts to the SDAI in order to build up and manage the Shared Data Base (SDB) of the Virtual Enterprise: the basic idea is that all the information, relevant to the operation of the V.E., are stored in a (logically) unique database, managed by the Technical Service which also controls the access to it, whereas the access rights of each node are defined by the Mgm Hub.

The whole set of information, being exchanged within ICIV, is split into two classes:

- Class S : information that are subjected to the Supervision of the Control Unit;

- Class N: information with No supervision.

It is a specific task of the Mgm Hub that of establishing criteria to sort out the information.

Basically:

- it is care of the Technical Service that of ensuring the consistency and the availability of the SDB;

- each node is provided with a mailbox in the system of the Technical Service;

- each information, sent by a node, is stored in the mailbox and sorted into either class $\mathrm{S}$ or class $\mathrm{N}$;

- class $S$ information are, then, stored within the SDB; a redirection to other nodes is care of the Technical Service;

- class N information are translated into the STEP file format, converted into the receiver system format and then forwarded to the receiver.

Whereas class $\mathrm{S}$ information have a "permanent" storage in the SDB, class $\mathrm{N}$ information are logged in a temporary buffer, periodically cleared, of the Technical Service system. 


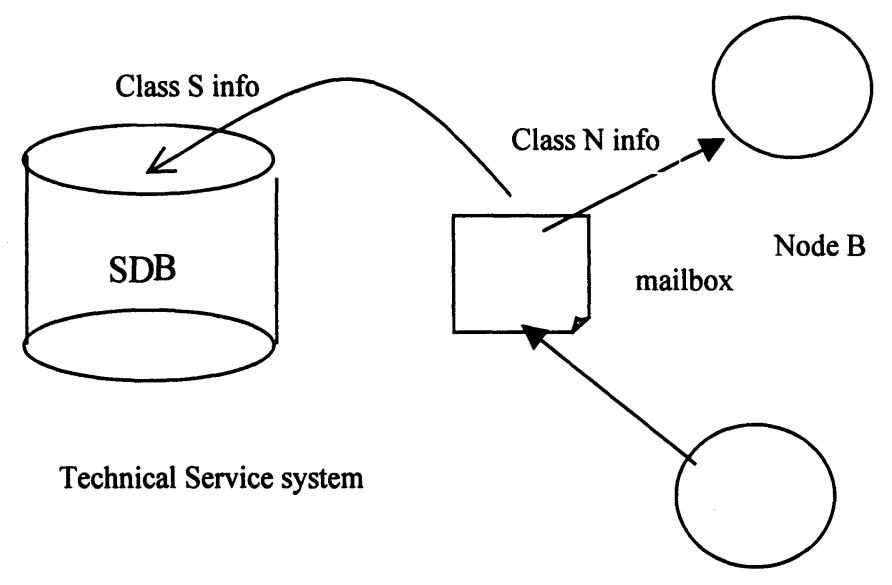

Figure 2 - Information exchange

Any other information exchanged directly between nodes, without the brokerage of the Control Unit, have no value for the V.E. (though they may have it for the nodes themselves).

This architecture of the information flow is adopted in order to support the control function of the Mgm Hub, which must coordinate independent operative units, often geographically dispersed, often changing over time.

Each node can keep using its own data storage practices and still be able to communicate with the other nodes; however, should a node be willing to switch to a STEP compliant data management, the efficiency of the above information flow would increase.

For SME's, the experience, gained in participating to such an arrangement, may encourage their direct adoption of the STEP standard, so that to become familiar with a tool which places them in a good strategic position when dealing with the global market.

\section{APPLICATIONS FORESEEN WITHIN PROJECT ICIV}

Within the end of year 2000, after the full development of the model, three test experiments are planned to be conducted:

- the first one will test the validity of some operational mechanisms in an environment of subcontractors;

- the second one will make up a V.E. aggregation of SME's in the field of Building \& Construction, around a military procurement; in fact, on the wave of CALS approach (4), the militaries are quite interested in the experiment;

- the third one will focus on the technologies adopted for the V.E., in particular on the simulation and Virtual Reality tools in the field of urban restructuring. 


\section{REFERENCES}

1. Bonfatti, F., Monari, P.D., Montanari, R., "Information flows and processes in a SME network"1996 - ESPRIT Program Project 20723 - PLENT (Planning Small-Medium Enterprise Network);

2. Bonfatti, F., Monari, P.D., Morini, A, "A new product data model for planning improvements at SMEs” - 1996 - ESPRIT Program Project 8224 - RUMS (a Ruled-based Manufacturing Modelling System);

3. Fowler J, "STEP for Data Management Exchange and Sharing", Technology Appraisals1995

4. Mc Adams A, "Using CALS to develop client-server solutions for reducing Health Care Logistic Cost", relazione presentata al Congresso CALS Europe 1998; 